Research Article

\title{
Ideals in quasi-ordered residuated system
}

\author{
Daniel A. Romano*
}

International Mathematical Virtual Institute, Kordunaška Street 6, 78000 Banja Luka, Bosnia and Herzegovina

(Received: 27 April 2021. Received in revised form: 3 June 2021. Accepted: 3 June 2021. Published online: 6 June 2021.)

(C) 2021 the authors. This is an open access article under the CC BY (International 4.0) license (www.creativecommons.org/licenses/by/4.0/).

\begin{abstract}
The concept of quasi-ordered residuated system was defined in 2018 by Bonzio and Chajda. The present author defined the notion of filters in quasi-ordered residuated system and described several different classes of filters in such systems. In this paper, the concept of pre-ideals and ideals in a residuated relational system ordered under a quasi-order are introduced and analyzed. Some important features of these classes of substructures in such relational systems are also shown. In addition, a congruence relation based on the concept of pre-ideal is constructed.
\end{abstract}

Keywords: relational system; residuated system; quasi-ordered residuated system; ideals in quasi-ordered residuated system.

2020 Mathematics Subject Classification: 08A02, 06A11, 06 B75.

\section{Introduction}

Some authors (for example, see [2]) associate the study of binary relational systems with Riguet's article [9]. Also, some authors (according to [2]) link Mal'cev's article [6] with the first attempts to research of relational systems into the algebraic frameworks. However, Maddux suggests that the paper [16] written by Tarski in 1941 is probably one of the first articles that relates to 'The calculus of relations' (see [5], page 438). The approach outlined in [16] is worked out in more detail in [17]. According to Madduox, the first definition of relation algebras appears in [4] (cited in [5], page 441).

In [2], Bonzio and Chajda introduced and analyzed the concept of 'residuated relational systems'. Before this paper, the residual relational system ordered under quasi-order was analyzed in Bonzio's doctoral dissertation [1]. This type of relational system was also considered in the paper [3]. Also, in the article [7] (see, page 165), a similar algebraic structure appears. In the articles [10-14], the present author dealt with filters in such systems.

In this article, we deal with ideals in quasi-ordered residuated systems. In Section 3, we analyze the right classes of the quasi-order relation in such systems. On the acquired properties of these classes are viewed as a guide for our intention to introduce the concept of ideals into a residuated relational system ordered under a quasi-order. In Subsection 4.1, we analyze the mutual relations of conditions through which we intend to define a class of ideals. In Subsections 4.2 and 4.3 , we introduce and analyze the class of pre-ideals and the class of ideals in quasi-ordered residuated systems. In Section 5 , a congruence relation $\theta_{J}$ based on a pre-ideal $J$ in such systems $\mathfrak{A}$ is constructed. Finally, it is shown that $\mathfrak{A} / \theta_{J}$ is also a quasi-ordered relational system.

\section{Preliminaries}

Definition 2.1 ([2], Definition 2.1). A residuated relational system is a structure $\mathfrak{A}=\langle A, \cdot, \rightarrow, 1, R\rangle$, where $\langle A, \cdot, \rightarrow, 1\rangle$ is an algebra of type $\langle 2,2,0\rangle$ and $R$ is a binary relation on A and satisfying the following properties:

(1) $(A, \cdot, 1)$ is a commutative monoid;

(2) $(\forall x \in A)((x, 1) \in R)$;

(3) $(\forall x, y, z \in A)((x \cdot y, z) \in R \Longleftrightarrow(x, y \rightarrow z) \in R)$.

We refer to the operation '.' as multiplication, to ' $\rightarrow$ ' as its residuum and to condition (3) as residuation. 
The basic properties for residuated relational systems are subsumed in the following theorem.

Theorem 2.1 ([2], Proposition 2.1). Let $\mathfrak{A}=\langle A, \cdot, \rightarrow, 1\rangle$ be a residuated system. Then

(4) $(\forall x, y \in A)(x \rightarrow y=1 \Longrightarrow(x, y) \in R)$;

(5) $(\forall x \in A)((x, 1 \rightarrow 1) \in R)$;

(6) $(\forall x \in A)((1, x \rightarrow 1) \in R)$;

(7) $(\forall x, y, z \in A)(x \rightarrow y=1 \Longrightarrow(z \cdot x, y) \in R)$;

(8) $(\forall x, y \in A)((x, y \rightarrow 1) \in R)$.

Recall that a quasi-order relation ' $\preccurlyeq$ ' on a set $A$ is a binary relation which is reflexive and transitive (some authors use the term pre-order relation).

Definition 2.2 ([2], Definition 3.1). A quasi-ordered residuated system is a residuated relational system $\mathfrak{A}=\langle A, \cdot, \rightarrow, 1, \preccurlyeq\rangle$, where $\preccurlyeq$ is a quasi-order relation in the monoid $(A, \cdot)$.

Example 2.1. Let $A=\{1, a, b, c, d\}$ and operations '.' and ' $\rightarrow$ ' defined on A as follows:

\begin{tabular}{|c|c|c|c|c|c|c|c|c|c|c|c|c|}
\hline & 1 & $a$ & $b$ & $c$ & $d$ & & $\rightarrow$ & 1 & $a$ & $b$ & $c$ & $d$ \\
\hline 1 & 1 & $a$ & $b$ & $c$ & $d$ & & 1 & 1 & $a$ & $b$ & $c$ & l \\
\hline o & $a$ & $a$ & $d$ & $c$ & $d$ & and & $a$ & 1 & 1 & $b$ & $c$ & $d$ \\
\hline$b$ & $b$ & $d$ & $b$ & $d$ & $d$ & and & $b$ & 1 & $a$ & 1 & $c$ & \\
\hline$c$ & $c$ & $c$ & $d$ & $c$ & $d$ & & $c$ & 1 & 1 & $b$ & 1 & $b$ \\
\hline$d$ & $d$ & $d$ & $d$ & $d$ & $d$ & & $d$ & 1 & 1 & 1 & 1 & 1 \\
\hline
\end{tabular}

Then $\mathfrak{A}=\langle A, \cdot, \rightarrow, 1\rangle$ is a quasi-ordered residuated system, where the relation ' $\preccurlyeq$ ' is defined as follows

$$
\preccurlyeq:=\{(1,1),(a, 1),(b, 1),(c, 1),(d, 1),(b, b),(a, a),(c, c),(d, d),(c, a),(d, a),(d, b),(d, c)\} .
$$

Example 2.2. Let $A=\{1, a, b, c, d, e\}$ and operations '’' and ' $\rightarrow$ ' defined on A as follows:

\begin{tabular}{l|llllll}
$\cdot$ & 1 & $a$ & $b$ & $c$ & $d$ & $e$ \\
\hline 1 & 1 & $a$ & $b$ & $c$ & $d$ & $e$ \\
$a$ & $a$ & $b$ & $a$ & $c$ & $d$ & $e$ \\
$b$ & $b$ & $b$ & $a$ & $c$ & $d$ & $e$ \\
$c$ & $c$ & $b$ & $c$ & $c$ & $d$ & $e$ \\
$d$ & $d$ & $d$ & $d$ & $d$ & $e$ & $e$ \\
$e$ & $e$ & $e$ & $e$ & $e$ & $e$ & $d$
\end{tabular}

\begin{tabular}{c|llllll}
$\rightarrow$ & 1 & $a$ & $b$ & $c$ & $d$ & $e$ \\
\hline 1 & 1 & $a$ & $b$ & $c$ & $d$ & $e$ \\
$a$ & 1 & 1 & $c$ & 1 & 1 & 1 \\
$b$ & 1 & $c$ & 1 & 1 & 1 & 1 \\
$c$ & 1 & $c$ & $c$ & 1 & 1 & 1 \\
$d$ & 1 & $d$ & $d$ & $d$ & 1 & $e$ \\
$e$ & 1 & $e$ & $e$ & $e$ & $d$ & 1
\end{tabular}

Then $\mathfrak{A}=\langle A, \cdot, \rightarrow, 1\rangle$ is a quasi-ordered residuated system, where the relation 'ß' is defined as follows

$\preccurlyeq:=\{(1,1),(a, 1),(b, 1),(c, 1),(d, 1),(e, 1),(a, a),(a, c),(a, d),(a, e),(b, b),(b, c),(b, d),(b, e),(c, c),(c, d),(c, e),(d, d),(e, e)\}$.

It should be noted that the elements a and $b$ are not comparable.

Example 2.3 ([8], Example 2.2). Any commutative residuated po-monoid (briefly, a CRPM) $(A, \cdot, \rightarrow, 1, \leqslant)$ is a quasi-ordered system.

Example 2.4. Let $\mathbb{R}$ be a field of real numbers. Define binary operations '.' and ' $\rightarrow$ ' on $A=[0,1] \subset \mathbb{R}$ by

$$
(\forall x, y \in[0,1])(x \cdot y:=\max \{0, x+y-1\}) \text { and } x \rightarrow y:=\min \{1,1-x+y\}) .
$$

Then, $A$ is a commutative monoid with the identity 1 and $\langle A, \cdot, \rightarrow, \leqslant, 1\rangle$ is a quasi-ordered residuated system.

The following proposition shows the basic properties of quasi-ordered residuated systems.

Proposition 2.1 ([2], Proposition 3.1). Let A be a quasi-ordered residuated system. Then

(9) $(\forall x, y, z \in A)(x \preccurlyeq y \Longrightarrow(x \cdot z \preccurlyeq y \cdot z \wedge z \cdot x \preccurlyeq z \cdot y))$;

(10) $(\forall x, y, z \in A)(x \preccurlyeq y \Longrightarrow(y \rightarrow z \preccurlyeq x \rightarrow z \wedge z \rightarrow x \preccurlyeq z \rightarrow y))$;

(11) $(\forall x, y \in A)(x \cdot y \preccurlyeq x \wedge x \cdot y \preccurlyeq y)$. 
In the rest of paper, by using the properties of the right classes of the quasi-order relation that exists in quasi-ordered residuated systems, we introduce and analyze the concept of ideals. First, we expose the properties of the right classes or the relation $\preccurlyeq$ in quasi-ordered residuated systems. We pay special attention to the connections between the multiplication operation and residual operation in such systems. Second, the idea of an ideal in a quasi-ordered residuated system $\langle A, \cdot, \rightarrow, \preccurlyeq\rangle$ will be based on a submonoid of the monoid $(A, \cdot)$ and it has some additional properties that will make this concept more complete. Additionally, we demonstrate several features of the so-designed substructure in quasi-ordered residuated systems.

\section{Right classes of quasi-order relations in a QRS}

Let $L(a)=\{y \in A: a \preccurlyeq y\}$ be the left class and $R(b)=\{x \in A: x \preccurlyeq b\}$ be the right class of the relation $\preccurlyeq$ generated by the elements $a$ and $b$ respectively. Then $R(1)=A$ by (2). In the following proposition, we give the basic properties of classes $R(b)(b \in A)$ :

Proposition 3.1. Let $a$ and $b$ be elements of $A$. The following holds

(12) $b \in R(b)$;

(13) If $\preccurlyeq$ is an antisymmetric relation, then

$$
1 \in R(b) \Longleftrightarrow b=1
$$

(14) $(\forall u, v \in A)((v \in R(b) \wedge u \preccurlyeq v) \Longrightarrow u \in R(b))$;

(15) $(\forall u, v \in A)(u \in R(b) \vee v \in R(b)) \Longrightarrow u \cdot v \in R(b))$;

(16) $a \preccurlyeq b \Longrightarrow R(a) \subseteq R(b)$;

(17) $R(a \cdot b) \subseteq R(a) \cap R(b)$; and

(18) Let us assume

(O) $\neg(1 \in R(b))$.

Then the following holds

$$
(\forall u, v \in A)(u \preccurlyeq v \Longrightarrow \neg(u \rightarrow v \in R(b))) .
$$

Proof. (12) It is clear that $b \in R(b)$ is valid due to the reflexivity of the relation $\preccurlyeq$.

(13) Let $b$ be an arbitrary element such that $1 \in R(b)$. Then $1 \preccurlyeq b$. Thus $b=1$ by (2) and antisymmetry of $\preccurlyeq$. The second part of this claim is obvious.

(14) Let $u, v \in A$ be arbitrary elements such that $v \in R(b)$ and $u \preccurlyeq v$. Then $v \preccurlyeq b \wedge u \preccurlyeq v$. Thus $u \preccurlyeq b$ by transitivity of $\preccurlyeq$. So, $u \in R(b)$.

(15) This claim is a direct consequence of condition (11). Let $u, v \in A$ be arbitrary elements such that $u \cdot v \in R(b)$. Then $u \preccurlyeq b$ and $v \preccurlyeq b$ by (11) and transitivity of $\preccurlyeq$.

(16) If $u \in R(a)$ then $u \preccurlyeq a \wedge a \preccurlyeq b$. Thus, $u \in R(b)$ by transitivity of $\preccurlyeq$.

(17) Let $u \in R(a \cdot b)$ be an arbitrary element. Then $u \preccurlyeq a \cdot b$. Thus $u \preccurlyeq a$ and $u \preccurlyeq b$ by (11) and transitivity of $\preccurlyeq$. So, $u \in R(a)$ and $u \in R(b)$. Therefore, $u \in R(a) \cap R(b)$.

(18) Suppose that $\neg(1 \in R(b))$. Let $u, v \in A$ be elements such that $u \preccurlyeq v$. Then $1 \preccurlyeq u \rightarrow v$ by (3). If we assume $u \rightarrow v \preccurlyeq b$, we have $1 \in R(b)$, which is a contradiction with the hypothesis. So, it has to be $\neg(u \rightarrow v \in R(b))$.

Example 3.1. Let $A$ be as in Example 2.1. Then, for example, take

$$
L(1)=\{1\}, L(a)=\{1, a\}, L(d)=A,
$$

and

$$
R(1)=A, R(a)=\{a, c, d\}, R(b)=\{b . d\}, R(c)=\{c, d\} \text { and } R(d)=\{d\} .
$$

It is obvious that the sets $R(a), R(b), R(c)$ and $R(d)$ satisfy the condition $(O)$. Also, we have $a \cdot b=d$ and

$$
\{d\}=R(d)=R(a \cdot b) \subseteq R(a) \cap R(b)=\{a, c, d\} \cap\{b, d\}
$$

which illustrates the claim (17). 
Proposition 3.2. If the right class $R(b)$ satisfies the condition (O), then the condition

(G) $(\forall u, v \in A)((\neg(u \rightarrow v \in R(b)) \wedge v \in R(b)) \Longrightarrow u \in R(b))$

implies condition (14).

Proof. Assume that $(\mathrm{O})$ and $(\mathrm{G})$ are valid formulas for $(R(b)$. Let $u, v \in A$ be arbitrary elements such that $u \preccurlyeq v$ and $v \in R(b)$. Then $\neg(u \rightarrow v \in R(b))$ by (18). Thus, we have $u \in R(b)$ by (G). So, the condition (14) is proven.

\section{Concepts of ideals}

\subsection{Analysis of conditions}

The properties of the right classes of the relation $\preccurlyeq$ provides a way for introducing the concept of ideals in a residuated relational system ordered under a quasi-order relation. Before that, we analyze the interrelation of the following formulas:

(J1) $(\forall u, v \in A)(u \in J \vee v \in J \Longrightarrow u \cdot v \in J)$;

(J2) $(\forall u, v \in A)((u \preccurlyeq v \wedge v \in J) \Longrightarrow u \in J)$; and

(J3) $(\forall u, v \in A)((\neg(u \rightarrow v \in J) \wedge v \in J) \Longrightarrow u \in J)$.

The condition (J1) implies that the subset $J$ is an ideal in the monoid $(A, \cdot)$. Therefore, it provides a link between the idea of this substructure and the internal binary operation in $A$. The condition (J2) implies that the subset $J$ is an ideal in the quasi-ordered ordered monoid $(A, \cdot)$. This condition will link the concept of ideals in a relational system $\mathfrak{A}$ with a quasi-order relation in $A$. The condition (J3) should involve the influence of the operation $\rightarrow$ into the substructure of the ideals in residuated relational systems ordered under quasi-order.

Proposition 4.1. $(J 2) \Longrightarrow(J 1)$.

Proof. The condition (J1) follows from the condition (J2) according to the (11).

Proposition 4.2. If for a proper subset J of a quasi-ordered residuated system (J2) holds, then the following is also valid

$$
(\forall u, v \in A)(u \preccurlyeq v \Longrightarrow \neg(u \rightarrow v \in J)) .
$$

Proof. Suppose (J2) holds. Let $u, v \in A$ be elements such that $u \preccurlyeq v$. Then $1 \preccurlyeq u \rightarrow v$ by (3). If we assume that $u \rightarrow v \in J$, then from $1 \preccurlyeq u \rightarrow v \in J$ by (J2) we get $1 \in J$. This gives $J=A$. This is in contradiction with the assumption that $J$ is a proper subset in $A$. Therefore, it has to be $\neg(u-v \in J)$.

Proposition 4.3. For a proper subset $J$ of a quasi-ordered residuated system $\mathfrak{A}$, it holds that $(J 3) \Longrightarrow(J 2)$.

Proof. Let $J$ be a proper subset of the monoid $(A, \cdot)$ in a quasi-ordered residuated system $\mathfrak{A}$ and let $u, v \in A$ be elements such that $u \preccurlyeq v$ and $v \in J$. Then $\neg(u \rightarrow v \in J)$ by Proposition 4.2. Now, from this and $v \in J$ follows $u \in J$ by (J3). So, the condition (J2) is proven.

Proposition 4.4. The condition ( $J 2)$ is equivalent to the condition

(J4) $(\forall u, v, z \in A)((u \preccurlyeq v \rightarrow z \wedge z \in J) \Longrightarrow u \cdot v \in J)$.

Proof. (J4) $\Longrightarrow(J 2)$. Assume that (J4) is valid and let $u, v \in A$ be arbitrary elements such that $u \preccurlyeq v$ and $v \in J$. Then $u \preccurlyeq 1 \rightarrow v$ and $v \in J$. Thus $u=u \cdot 1 \in J$ by (J4). So, the condition (J2) is proven.

$(J 2) \Longrightarrow(J 4)$. Suppose (J2) holds and let $u, v, z \in A$ be arbitrary elements such that $u \preccurlyeq v \rightarrow z$ and $z \in J$. Then $u \cdot v \preccurlyeq z$ and $z \in J$ by (3). Thus $u \cdot v \in J$ by (J2). So, the condition (J4) is proven.

Proposition 4.5. Let $J$ be a consistent subset of the monoid $(A, \cdot)$ in a quasi-ordered residuated system $\mathfrak{A}=\langle A, \cdot, \rightarrow, 1\rangle$. Then $(J 2) \Longrightarrow(J 3)$.

Proof. Let $u, v \in A$ be arbitrary elements such that $\neg(u \rightarrow v \in J)$ and $v \in J$. From $u \rightarrow v \preccurlyeq u \rightarrow v$ follows $(u \rightarrow v) \cdot u \preccurlyeq v$ by (3). Thus $(u \rightarrow v) \cdot u \in J$ by (J2). From this follows $u \rightarrow v \in J \vee u \in J$ by consistency of $J$ in $(A, \cdot)$. Since the first option is impossible by hypothesis, it has to be $u \in J$. Therefore, the implication (J3) is proven. 
Remark 4.1. The conditions (J2) and (J3) are not equivalent in the general case. If we choose to determine the concept of ideals by condition (J3), then the defined substructure in a quasi-ordered residuated system satisfy the conditions (J1) and (J2). In this case, the right classes, in the general case, are not ideals. If we choose to define the concept of ideals by condition (2), then we have at least two types of ideals in these systems: ideals of type 1 - the class of ideals defined by condition (J2), and ideals of type 2 - the class of ideals determined by condition (J3). The second type of ideal, determined by the condition (J3), are at the same time the first kind of ideal, because $(J 3) \Longrightarrow(J 2)$.

Proposition 4.6. The condition (J3) is equivalent to the condition

(J5) $(\forall u, v \in A)((\neg(u \in J) \wedge v \in J) \Longrightarrow u \rightarrow v \in J)$.

Proof. (J3) $\Longrightarrow$ (J5). Suppose that formula (J3) is valid and let $u, v \in A$ be elements such that $u \notin J$ and $v \in J$. If we assume that $u \rightarrow v \notin J$ holds, then it would be $u \in J$ according to (J3). The resulting contradiction refutes the assumption. So it has to be $u \rightarrow v \in J$.

(J5) $\Longrightarrow(\mathrm{J} 3)$. Conversely, let (J5) be valid and let the elements $u, v \in A$ be such that $u \rightarrow v \notin J$ and $v \in J$. If we assume that $u \notin J$ is valid we would have $u \rightarrow v \in J$ according to (J5). This contradicts the hypothesis. So it must be $u \in J$.

Corollary 4.1. Let $J$ be an ideal of q quasi-ordered residuated system $\mathfrak{A}$ such that $1 \notin J$. Then the following holds

$$
(\forall v \in A)(v \in J \Longrightarrow 1 \rightarrow v \in J) .
$$

Proof. The claim follows directly from Proposition 4.6.

\subsection{Concept of pre-ideals}

As usual in any algebraic structure $\mathfrak{A}, A$ is one of the ideals of structure $\mathfrak{A}$. By depending on our orientation about the determination of the notion of ideals in a residuated relational system ordered $\mathfrak{A}$ under a quasi-order by the properties of the right classes $R(a)(a \in A)$ of the relation $\preccurlyeq$ generated by elements of the monoid $(A, \cdot)$, we introduce the concept of the pre-ideal in the system $\mathfrak{A}$ in the following way.

Definition 4.1. Let $\mathfrak{A}=\langle A, \cdot, \rightarrow, 1, \preccurlyeq\rangle$ be a quasi-ordered residuated system. For a subset $J$ of the set $A$ we say that it is an pre-ideal in $\mathfrak{A}$ if the condition (J2) is valid.

The class $R(b)(b \in A)$ gives pre-ideals in $\mathfrak{A}$. Furthermore, the sets $\emptyset$ and $A$ are trivial pre-ideals in $\mathfrak{A}$. Therefore, the family $\mathfrak{p} \mathfrak{J}(A)$ of all pre-ideals in a quasi-ordered residuated system $\mathfrak{A}$ is non-empty.

Theorem 4.1. The family $\mathfrak{p} \mathfrak{J}(A)$ of all pre-ideals in a quasi-ordered residuated system $\mathfrak{A}$ forms a completely lattice.

Proof. Let $\left\{J_{i}\right\}_{i \in I}$ be a family of pre-ideals in a quasi-ordered residuated system $\mathfrak{A}$.

(i) Let $u \preccurlyeq v$ and $v \in \bigcup_{i \in I} J_{i}$. Then there exists an index $j \in I$ such that $v \in J_{j}$. Thus $u \in J_{j} \subseteq \bigcup_{i \in I} J_{i}$ by (J2). So, $\bigcup_{i \in I} J_{i}$ is a pre-ideal in $\mathfrak{A}$.

(ii) Let $\mathfrak{B}$ be the family of all pre-ideals in $\mathfrak{A}$ contained in $\bigcap_{i \in I} J_{i}$. Then $\cup \mathfrak{B}$ is the maximal pre-ideal in $\mathfrak{A}$ contained in $\bigcap_{i \in I} J_{i}$ according to (i) of this proof.

(iii) If we put $\sqcup_{i \in I} J_{i}=\bigcup_{i \in I} J_{i}$ and $\sqcap_{i \in I}=\cup \mathfrak{B}$, then $(\mathfrak{J}(A), \sqcup, \sqcap)$ is a completely lattice.

Corollary 4.2. Let $\mathfrak{A}$ be a quasi-ordered residuated system and $T$ be a non-empty subset of $A$. Then $T^{U}=\bigcup_{b \in T} R(b)$ is a pre-ideal in $\mathfrak{A}$.

Proof. Since $R(b)$ is a pre-ideal in $A$, then $T^{U}=\bigcup_{b \in T} R(b)$ is a pre-ideal in $\mathfrak{A}$ by the first part of the proof of Theorem 4.1.

This type of pre-ideal is called normal pre-ideals.

Theorem 4.2. If $\left\{J_{k}\right\}_{k \in K}$ is a family of normal pre-ideals in $\mathfrak{A}$, then $\bigcup_{k \in K} J_{k}$ is also a normal pre-ideal in $\mathfrak{A}$.

Proof. Since $J_{k}$ is a normal pre-ideal in $A$, there exist a subset $T_{k}$ such that $J_{k}=T_{k}^{U}=\bigcup_{t \in T_{k}} R(t)$. The claim of this theorem is based on the equality $\bigcup_{k \in K} T_{k}^{U}=\left(\bigcup_{k \in K} T_{k}\right)^{U}$.

Theorem 4.3. Every pre-ideal $J$ in a quasi-ordered residuated system $\mathfrak{A}$ is a normal pre-ideal.

Proof. Let $J$ be a pre-ideal in $\mathfrak{A}$ and let $u \in H$. Then $u \in R(u)$ by (12). Thus $J \subseteq \bigcup_{b \in J} R(b)$.

Conversely, let $u \in \bigcup_{b \in J} R(b)$. Then there exists an element $b \in J$ such that $u \in R(b)$. This means $u \preccurlyeq b \wedge b \in J$. Thus $u \in J$ by (J2). So, $\bigcup_{b \in J} \subseteq J$. 
Remark 4.2. The intersection of two normal pre-ideals need not to be a pre-ideal. If $J_{1}$ and $J_{2}$ are pre-ideals in $\mathfrak{A}$, then there are subsets $T_{1}$ and $T_{2}$ in $A$, such that $J_{1}=T_{1}^{U}$ and $J_{2}=T_{2}^{U}$. Thus $\left.J_{1} \cap J_{2}=T_{1}^{U} \cap T_{2}^{U}=\left(\bigcup_{t \in T_{1}} R(t)\right) \cap \bigcup_{s \in T_{2}} R(s)\right)=$ $\bigcup_{t \in T_{1}} \bigcup_{s \in T_{2}}(R(t) \cap R(s)) \supseteq \bigcup_{t \in T_{1}} \bigcup_{s \in T_{2}} R(t \cdot s)$ by (17).

Example 4.1. Let $A$ be as in Example 2.2. Then the subsets $R(1)=A, R(a)=\{a, c, d, e\}, R(b)=\{b\}, R(c)=\{a, b, c\}$, $R(d)=\{a, b, c, d\}$ and $R(e)=\{a, b, c, e\}$ are pre-ideals in the quasi-ordered residuated system $\langle A, \cdot, \rightarrow, 1\rangle$. For example, for incomparable elements a and $b$, we have $\{a, b\}^{U}=R(a) \cup R(b)=A$ which illustrates the Corollary 4.2.

\subsection{Concept of ideals}

Definition 4.2. Let $\mathfrak{A}=\langle A, \cdot, \rightarrow, 1, \preccurlyeq\rangle$ be a quasi-ordered residuated system. For a subset $J$ of the set $A$ we say that it is an ideal in $\mathfrak{A}$ if $J=A$ or the condition (J3) is valid.

Proposition 4.7. Every ideal J in a quasi-ordered residuated system $\mathfrak{A}$ is a pre-ideal in $\mathfrak{A}$.

Proof. The concept of ideal in a quasi-ordered residuated system is defined by $J=A$ or the subset $J$ satisfies the condition (J3). If $J=A$, then $J$ is a pre-ideal in $\mathfrak{A}$, according to Definition 4.1. If the ideal $J$ in $\mathfrak{A}$ is a proper subset of $A$, then $J$ is a pre-ideal in $\mathfrak{A}$, according to Proposition 4.3.

The class $R(b)$ satisfying the conditions $(\mathrm{O})$ and $(\mathrm{G})$ is an ideal in $\mathfrak{A}$. Furthermore, the sets $\emptyset$ and $A$ are trivial ideals in $\mathfrak{A}$. Therefore, the family $\mathfrak{J}(A)$ of all ideals in a quasi-ordered residuated system $\mathfrak{A}$ is not empty.

Theorem 4.4. The family $\mathfrak{J}(A)$ of all ideals in a quasi-ordered residuated system $\mathfrak{A}$ forms a completely lattice.

Proof. Let $\left\{J_{i}\right\}_{i \in I}$ be a family of ideals in a quasi-ordered residuated system $\mathfrak{A}$. For the sake of simplicity in making conclusions, we assume that ideals in the quasi-ordered system $\mathfrak{A}$ are proper ideals.

(i) Let $\neg\left(u \rightarrow v \in \bigcup_{i \in I} J_{i}\right)$ and $v \in \bigcup_{i \in I} J_{i}$. Then $(\forall i \in I) \neg\left(u \rightarrow v \in J_{i}\right)$ and there exists an index $j \in I$ such that $v \in J_{j}$. Thus $u \in J_{j} \subseteq \bigcup_{i \in I} J_{i}$ by (J3). So, $\bigcup_{i \in I} J_{i}$ is an ideal in $\mathfrak{A}$.

(ii) Let $\mathfrak{B}$ be the family of all ideals in $\mathfrak{A}$ contained in $\bigcap_{i \in I} J_{i}$. Then $\cup \mathfrak{B}$ is the maximal ideal in $\mathfrak{A}$ contained in $\bigcap_{i \in I} J_{i}$ according to (i) of this proof.

(iii) If we put $\sqcup_{i \in I} J_{i}=\bigcup_{i \in I} J_{i}$ and $\sqcap_{i \in I}=\cup \mathfrak{B}$, then $(\mathfrak{J}(A), \sqcup, \sqcap)$ is a completely lattice.

Corollary 4.3. For each subset $B$ in a quasi-ordered residuated system $\mathfrak{A}$ there is the maximal ideal in $\mathfrak{A}$ included in $B$.

Proof. The proof of this assertion follows directly from the second part of the proof of Theorem 4.4.

Corollary 4.4. For any element a in a quasi-ordered residuated system $\mathfrak{A}$ there exists the maximal ideal $M_{a}$ such that $\neg\left(a \in M_{a}\right)$.

Proof. The claim follows from Corollary 4.3 if we take $B=A \backslash\{a\}$.

Example 4.2. Let $A$ be as in Example 2.1. Then, the subset $R(a)=\{a, c, d\}$ is an ideal in $\mathfrak{A}$. Indeed, for the elements $1, b \in A$, we have $1 \notin R(a)$ and $b \notin R(a)$. By direct verification, the following properties can be easily proved:

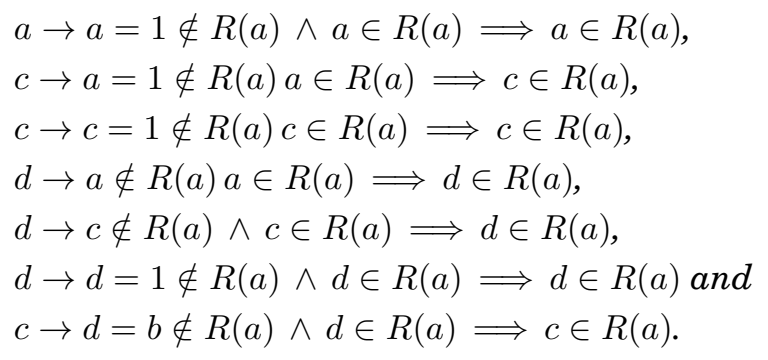

Analogously, it can be verified that the set $R(b)$ is an ideal in $\mathfrak{A}$. 


\section{A construction of congruences}

We recall that any quasi-order relation on a set $A$ generates an equivalence relation as follows

$$
\left(C_{\preccurlyeq}\right)(\forall x, y \in A)\left((x, y) \in \theta_{\preccurlyeq} \Longleftrightarrow(x \preccurlyeq y \wedge y \preccurlyeq x)\right) .
$$

The internal binary operations in $A$ are compatible with the relation $\theta_{\preccurlyeq}$ according to (9) and (10).

The equivalence relation $\theta_{\preccurlyeq}$, described by $\left(C_{\preccurlyeq}\right)$, suggests that we can form the concept of congruences on a quasi-ordered residuated system analogously.

Definition 5.1. An equivalence relation $\theta$ on a quasi-ordered residuated system $\mathfrak{A}=\langle A, \cdot, \rightarrow, 1, \preccurlyeq\rangle$ is a congruence on $\mathfrak{A}$ if the the following conditions hold

(C1) $(\forall x, y, z \in A)((x, y) \in \theta \Longrightarrow((x \cdot z, y \cdot z) \in \theta \wedge(z \cdot x, z \cdot y) \in \theta))$;

(C2) $(\forall x, y, z \in A)((x, y) \in \theta \Longrightarrow((x \rightarrow z, y \rightarrow z) \in \theta \wedge(z \rightarrow x, z \rightarrow y) \in \theta))$.

Note 5.1. Due to the commutativity of the multiplication operation in A, in implication (C1) it is sufficient to search for one of two requirements.

In what follows, we write the relation of the congruence $C_{\preccurlyeq}$ as $\equiv_{\preccurlyeq}$.

Let $\langle A, \cdot, \rightarrow, \preccurlyeq, 1\rangle$ and $\left\langle B,,^{\prime}, \rightarrow^{\prime}, \preccurlyeq^{\prime}, 1^{\prime}\right\rangle$ be two quasi-ordered residuated systems. A mapping $f: A \longrightarrow B$ is a homomorphism between quasi-ordered residuated systems if the following condition holds

$$
(\forall u, v \in A)\left(f(1) \equiv_{\preccurlyeq^{\prime}} 1^{\prime} \wedge f(u \cdot v) \equiv_{\preccurlyeq^{\prime}} f(u) \cdot^{\prime} f(v) \wedge f(u \rightarrow v) \equiv_{\preccurlyeq^{\prime}} f(u) \rightarrow^{\prime} f(v)\right) .
$$

A homomorphism $f$ is a monotone if it holds that

$$
(\forall u, v \in A)\left(u \preccurlyeq v \Longrightarrow f(u) \preccurlyeq^{\prime} f(v)\right) .
$$

Example 5.1. Without much difficulties, one can check that the relation Kerf $\left.:=\{u, v) \in A \times A: f(u) \equiv \preccurlyeq^{\prime} f(v)\right\}$, where $f$ is a monotone homomorphism, is a congruence on A. Indeed:

Let $u, v, z \in A$ be elements such that $(u, v) \in \operatorname{Kerf}$. Then $f(u) \equiv_{\preccurlyeq^{\prime}} f(v)$. Thus

$f(x \cdot z) \equiv \preccurlyeq^{\prime} f(u) \cdot \cdot^{\prime} f(z) \preccurlyeq^{\prime} f(v) \cdot \cdot^{\prime} f(z) \preccurlyeq^{\prime} f(v \cdot z)$ by (9) and

$f(v \cdot z) \equiv \preccurlyeq^{\prime} f(v) \cdot^{\prime} f(z) \preccurlyeq^{\prime} f(u) \cdot^{\prime} f(z) \equiv \preccurlyeq^{\prime} f(u \cdot z)$ by $(9)$.

Hence $(u \cdot z, v \cdot z) \in \operatorname{Kerf}$.

Let $u, v, z \in A$ be elements such that $(u, v) \in K$ Kerf. Then $f(u) \equiv \preccurlyeq^{\prime} f(v)$. This means $f(u) \preccurlyeq^{\prime} f(v)$ and $f(v) \preccurlyeq^{\prime} f(u)$. Thus $f(u \rightarrow z) \equiv \preccurlyeq^{\prime} f(u) \rightarrow^{\prime} f(z) \preccurlyeq^{\prime} f(v) \rightarrow^{\prime} f(z) \preccurlyeq^{\prime} f(v \rightarrow z)$ by $(10)$ and

$f(v \rightarrow z) \equiv_{\preccurlyeq^{\prime}} f(v) \rightarrow^{\prime} f(z) \preccurlyeq^{\prime} f(u) \rightarrow^{\prime} f(z) \equiv_{\preccurlyeq^{\prime}} f(u \rightarrow z)$ by $(10)$.

Hence $(u \rightarrow z, v \rightarrow z) \in \operatorname{Ker} f$. The second implication can be proved analogously to the previous one.

Theorem 5.1. The family $\mathfrak{C}(A)$ of all congruences on a quasi-ordered residuated system $\mathfrak{A}$ forms a complete lattice.

Proof. Let $\left\{\theta_{i}\right\}_{i \in I}$ be a family of congruences on $\mathfrak{A}$. It can be proved that $\bigcap_{i \in I} \theta_{i}$ is a congruence on $\mathfrak{A}$ by a direct check. Let $\mathfrak{X}$ be a family of congruences on $\mathfrak{A}$ containing $\bigcup_{i \in I} \theta_{i}$. Then $\cap \mathfrak{X}$ is the minimal congruence on $\mathfrak{A}$ containing $\bigcup_{i \in I} \theta_{i}$ by the first part of this proof. If we put $\sqcup_{i \in I} \theta_{i}=\cap \mathfrak{X}$ and $\sqcap_{i \in I} \theta_{i}=\bigcap_{i \in I} \theta_{i}$, then $(\mathfrak{C}(A), \sqcup, \sqcap)$ is a complete lattice.

In the following theorem, we show a construction of congruence on a quasi-ordered residuated system $A$ for a given pre-ideal $J$ of $A$.

Theorem 5.2. If $J$ is a pre-ideal of a quasi-ordered residuated system $\mathfrak{A}=\langle A, \cdot, \rightarrow, 1, \preccurlyeq\rangle$ then the relation $\theta_{J}$, defined by $\left(C_{J}\right)(\forall x, y \in A)\left((x, y) \in \theta_{J} \Longleftrightarrow(\exists a \in J)(a \cdot x \preccurlyeq y \wedge a \cdot y \preccurlyeq x)\right)$,

is a convex congruence on A.

Proof. It is obvious that $\theta_{J}$ is a reflexive and symmetric relation. Let $x, y, z \in A$ be such that $(x, y) \in \theta_{J}$ and $(y, z) \in \theta_{J}$. Then there exist elements $a \in J$ and $b \in J$ such that $a \cdot x \preccurlyeq y \wedge a \cdot y \preccurlyeq x$ and $b \cdot y \preccurlyeq z \wedge b \cdot z \preccurlyeq y$. Thus $(a \cdot b) \cdot x=b \cdot(a \cdot x) \preccurlyeq b y \preccurlyeq z$ and $(a \cdot b) \cdot z=a \cdot(b \cdot z) \preccurlyeq a \cdot y \preccurlyeq z$. As $a \cdot b \in J$ by $(\mathrm{J} 1)$, this means $(x, z) \in \theta_{J}$. This proves that $\theta_{J}$ is an equivalence relation on the system $\mathfrak{A}$. 
Let $x, y, z \in A$ be elements such that $(x, y) \in \theta_{J}$. Then there exists an element $a \in J$ such that $a \cdot x \preccurlyeq y$ and $a \cdot y \preccurlyeq x$. Thus $a \cdot(x \cdot z) \preccurlyeq x \cdot z$ and $a \cdot(y \cdot z) \preccurlyeq x \cdot z$ according to (9). Hence $(x \cdot z, y \cdot z) \in \theta_{J}$. It is shown that the relation $\theta_{J}$ is compatible with the operation '.' in $A$.

Let $x, y, z \in A$ be arbitrary elements such that $(x, y) \in \theta_{J}$. Then there exists an element $a \in J$ such that $a \cdot x \preccurlyeq y$ and $a \cdot y \preccurlyeq x$. Thus

$$
a \preccurlyeq x \rightarrow y \preccurlyeq(y \rightarrow z) \rightarrow(x \rightarrow z) \text { and } a \preccurlyeq y \rightarrow x \preccurlyeq(x \rightarrow z) \rightarrow(y \rightarrow z)
$$

by (12). Hence

$$
a \cdot(y \rightarrow z) \preccurlyeq x \rightarrow z \text { and } a \cdot(x \rightarrow z) \preccurlyeq y \rightarrow z .
$$

This means $(x \rightarrow z, y \rightarrow z) \in \theta_{J}$.

On the other hand, from $a \cdot x \preccurlyeq y$ and $a \cdot y \preccurlyeq x$ follows $z \rightarrow a \cdot x \preccurlyeq z \rightarrow y$ and $z \rightarrow a \cdot y \preccurlyeq z \rightarrow x$ according to (10). From here we get $a \cdot(z \rightarrow x) \preccurlyeq z \rightarrow y$ and $a \cdot(z \rightarrow y) \preccurlyeq z \rightarrow x$ according to (13). So, we get $(z \rightarrow x, z \rightarrow y) \in \theta_{J}$. Therefore, the relation $\theta_{J}$ is a congruence on $\mathfrak{A}$.

The relation $\theta_{J}$, constructed in this way, is a convex relation to $A$. Let $x, y, z \in A$ be such that $x \preccurlyeq y \preccurlyeq z$ and $(x, z) \in \theta_{J}$. Then there exists an element $a \in J$ such that $a \cdot x \preccurlyeq z$ and $a \cdot z \preccurlyeq x$. From $y \preccurlyeq z$ it follows $a \cdot y \preccurlyeq z \cdot z$ by (9). Since $a \cdot z \preccurlyeq x$, we have $a \cdot y \preccurlyeq x$. On the other hand, the following $a \cdot x \preccurlyeq x \preccurlyeq y$ holds by (11). Hence $(x, y) \in \theta_{J}$.

Note 5.2. It is obvious that $\theta_{\preccurlyeq} \cap(J \times J) \subseteq \theta_{J}$. For example, we can take $a=y$. Then we have $a \cdot x=x \cdot y \preccurlyeq y$ and $a \cdot y=y \cdot y \preccurlyeq y \preccurlyeq x$ according to (11).

Remark 5.1. The condition $\left(C_{J}\right)$ is equivalent to the condition

$$
\left.(x, y) \in \theta_{J} \Longleftrightarrow(\exists a \in J)(x \rightarrow y \in L(a) \wedge y \rightarrow x \in L(a))\right)
$$

where $L(a)=\{u \in A: a \preccurlyeq u\}$ is the left class of the relation $\preccurlyeq$ generated by the element $a$.

Remark 5.2. Let us note that in the previous theorem, for given elements $x, y \in A$ such that $(x, y) \in \theta_{J}$, the element a $\in J$, which appears in the definition $\left(C_{J}\right)$, is not unique. Indeed, for every $a^{\prime} \preccurlyeq a$, the following are valid $a^{\prime} \in J$ (according to (J2)) and $a^{\prime} \cdot x \preccurlyeq a \cdot x \preccurlyeq y$ and $a^{\prime} \cdot y \preccurlyeq a \cdot y \preccurlyeq x$ by (9).

The importance of a congruence relation $\theta$ on a quasi-ordered residuated system $\mathfrak{A}$ is justified by the fact that the quotient $A / \theta$ turns naturally into an ordered set. It is commonly known that if $(A, \preccurlyeq)$ is a quasi-ordered set and $\theta$ is an equivalence relation on $A$, then the relation $\leqq$, defined by $(\forall x, y \in A)\left([x]_{\theta} \leqq[y]_{\theta} \Longleftrightarrow x \preccurlyeq y\right)$ is an order relation on $A / \theta$. Let us define operations ‘ $\odot$ ’ and ‘ $\rightrightarrows$ ’ as

$$
\begin{gathered}
(\forall x, y \in A)\left([x]_{\theta} \odot[y]_{\theta}=[x \cdot y]_{\theta}\right) \text { and } \\
(\forall x, y \in A)\left([x]_{\theta} \rightrightarrows[y]_{\theta}=[x \rightarrow y]_{\theta}\right) .
\end{gathered}
$$

Theorem 5.3. Let $\mathfrak{A}=\langle A, \cdot, \rightarrow, 1, \preccurlyeq\rangle$ be a quasi-ordered relational system and let $\theta$ be a congruence on $\mathfrak{A}$. Then

$$
\left\langle A / \theta, \odot, \rightrightarrows,[1]_{\theta}, \leqq\right\rangle
$$

is a quasi-ordered residuated system.

Proof. It is easy to verify that the operation $\odot$ is consistent with the order relation $\leqq$ in $A / \theta$. Let $x, y, z \in A$ be such that $[x]_{\theta} \leqq[y]_{\theta}$. This means $x \preccurlyeq y$. Then $x \cdot z \preccurlyeq y \cdot z$ by (9). Thus $[x \cdot z]_{\theta} \leqq[y \cdot z]_{\theta}$. Hence $[x]_{\theta} \odot[z]_{\theta} \leqq[y]_{\theta} \odot[z]_{\theta}$.

Without difficulty, it is verified that $\left(A / \theta, \odot,[1]_{\theta}\right)$ is a monoid relative to the operation ' $\odot$ '.

Since $(\forall x \in A)(x \preccurlyeq 1)$, we have $(\forall x \in A)\left([x]_{\theta} \leqq[1]_{\theta}\right)$. This shows that condition (2) is valid for the system $\langle A / \theta, \odot, \rightrightarrows$ $\left.,[1]_{\theta}, \leqq\right\rangle$.

Let $x, y, z \in A$ be elements such that $[x]_{\theta} \odot[y]_{\theta} \leqq[z]_{\theta}$. Then $[x \cdot y]_{\theta} \leqq[z]_{\theta}$. This means $x \cdot y \preccurlyeq z$. Thus $x \preccurlyeq y \rightarrow z$ by (3). This last inequality gives $[x]_{\theta} \leqq[y \rightarrow z]_{\theta}$. Hence $[x]_{\theta} \leqq[y]_{\theta} \rightrightarrows[z]_{\theta}$ by definition of residuum in the system $\left\langle A / \theta, \odot, \rightrightarrows,[1]_{\theta}, \leqq\right\rangle$. Therefore, formula (3) is valid for the system $\left\langle A / \theta, \odot, \rightrightarrows,[1]_{\theta}, \leqq\right\rangle$.

Because of Theorem 5.2 and Theorem 5.3, we have:

Theorem 5.4. Let $J$ be a pre-ideal of a quasi-ordered residuated system $\langle A, \cdot, \rightarrow, 1, \preccurlyeq\rangle$. Then the quasi-ordered residuated system $\left\langle A / \theta_{J}, \odot, \rightrightarrows,[1]_{\theta_{J}}, \leqq\right\rangle$ can be constructed. In addition, natural mapping $\pi: A \longrightarrow A / \theta_{J}$ is a monotone surjective homomorphism of quasi-ordered residuated systems. 
Proof. For a given ideal $J$ in a quasi-ordered residuated system $\mathfrak{A}$, the congruence $\theta_{J}$ can be constructed, according to Theorem 5.2. The proof follows directly from Theorem 5.3. It is obvious that the correspondence $\pi: A \longrightarrow A / \theta_{J}$, determined in the following way: $(\forall x \in A)\left(\pi(x):=[x]_{\theta_{J}}\right)$, is a surjective function.

As a conclusion of this section, we can express the following theorem:

Theorem 5.5. Let $f: A \longrightarrow A^{\prime}$ be a monotone homomorphism between quasi-ordered residuated systems. Then there exists the injective homomorphism

$$
g:\left\langle A / \operatorname{Kerf}, \odot, \rightrightarrows,[1]_{\text {Kerf }}, \leqq\right\rangle \longrightarrow\left\langle A^{\prime}, .^{\prime}, \rightarrow^{\prime}, \preccurlyeq^{\prime}, 1^{\prime}\right\rangle
$$

such that $f=g \circ \pi$.

Proof. Kerf is a congruence on a quasi-ordered residuated system $\mathfrak{A}$ as shown in Example 5.1 which allows us to construct a quasi-ordered residuated system $\left\langle A / \operatorname{Kerf}, \odot, \rightrightarrows,[1]_{K e r f}, \leqq\right\rangle$. Let us determine the function $g: A / \operatorname{Kerf} \longrightarrow A^{\prime}$ as follows $\left(\forall[x]_{\text {Kerf }} \in A / \operatorname{Kerf}\right)\left(g\left([x]_{\text {Kerf }}\right):=f(x)\right)$.

Let $x, y \in A$ be such that $[x]_{\text {Kerf }}=[y]_{\text {Kerf } f}$. Then $f(x) \equiv_{\preccurlyeq^{\prime}} f(y)$. This shows that $g$ is a function. Conversely, if

$$
f(x):=g\left([x]_{\text {Kerf }}\right) \equiv \preccurlyeq^{\prime} g\left([y]_{\text {Kerf }}\right):=f(y)
$$

holds, then $[x]_{\text {Kerf }}=[y]_{\text {Kerf } f}$. This shows that $g$ is an injective function.

Since the following holds

$$
\begin{gathered}
g\left([x]_{\text {Kerf }} \odot[y]_{\text {Kerf }}\right) \equiv_{\preccurlyeq^{\prime}} g\left([x \cdot y]_{\text {Kerf }}\right):=f(x \cdot y)=^{\prime} f(x) \cdot^{\prime} f(y):=g\left([x]_{\text {Kerf }}\right) \cdot^{\prime} g\left([y]_{\text {Kerf }}\right), \text { and } \\
g\left([x]_{\text {Kerf }} \rightrightarrows[y]_{\text {Kerf }}\right) \equiv_{\preccurlyeq^{\prime}} g\left([x \rightarrow y]_{\text {Kerf }}\right):=f(x \rightarrow y)=^{\prime} f(x) \rightarrow^{\prime} f(y):=g\left([x]_{\text {Kerf }}\right) \rightarrow^{\prime} g\left([y]_{\text {Kerf }}\right),
\end{gathered}
$$

we conclude that $g$ is homomorphism.

\section{Final observations}

The algebraic structure of a residuated relational system ordered under a quasi-order relation $\mathfrak{A}=\langle A, \cdot, \rightarrow, 1, \preccurlyeq\rangle$ implies the existence (and their specific interdependence $x \cdot y \preccurlyeq z \Longleftrightarrow x \preccurlyeq y \rightarrow z$ ) of two internal binary operations, a multiplication '.' and a residuum ' $\rightarrow$ ', on a given set $A$ such that $(A, \cdot, 1)$ is a monoid. In the last decade, this algebraic structure is the focus of some researchers (for example, see $[1-3,7,11]$ ). While other researchers were interested in linking this structure with possible induced algebraic systems, the present author is interested in the substructures of these systems. In the articles [11-14], the concept of filters in these systems was the subject of the study. In this article, the possibility of introducing the concept of ideals in such systems is discussed. It is quite natural to pose the question: Is it possible to introduce the class of ideals in quasi-ordered residuated systems in the way different from the one chosen in this paper?

As a final comment, we offer the following conclusion: In any quasi-ordered residuated system $\mathfrak{A}$ there is a correspondence between the lattice $p \mathfrak{J}(A)$ of all pre-ideals of $\mathfrak{A}$ and the lattice $\mathfrak{C}(A)$ of all congruences on $\mathfrak{A}$ such that each pre-ideal $J$ of $\mathfrak{A}$ is associated with the congruence $\theta_{J}$.

\section{References}

[1] S. Bonzio, Algebraic Structures from Quantum and Fuzzy Logics, Ph.D. Thesis, Università degli Studi di Cagliari, Cagliari, 2015.

[2] S. Bonzio, I. Chajda, Residuated relational systems, Asian-Eur. J. Math., 11 (2018) Art\# 1850024.

[3] I. Chajda, J. Kühr, H. Länger. Relatively residuated lattices and posets, Math. Slovaca 70 (2020) 239-250.

[4] B. Jónsson, A. Tarski. Representation problems for relation algebras (Abstract 89T), Bull. Amer. Math. Soc. 54 (1948) pp. 80 and pp. 1192.

[5] R. D. Maddux, The origin of relation algebras in the development and axiomatization of the calculus of relations, Studia Logica $\mathbf{5 0}$ (1991) $421-455$.

[6] A. I. Malćev, On the general theory of algebraic systems, Mat. Sb. 35 (1954) 3-20 (In Rusian).

[7] A. M. Radzikowska, E. E. Kerre, Fuzzy information relations and operators: An algebraic approach based on residuated lattices, In: H. de Swart, E. Orlowska, G. Schmidt, M. Roubens (Eds.), Theory and Applications of Relational Structures as Knowledge Instruments II, Springer, Berlin, 2006, pp. $162-184$.

[8] J. G. Raftery, Order algebraizable logics, Ann. Pure Appl. Logic 164 (2013) 251-283.

[9] J. Riguet, Relations binaires, fermetures, correspondances de Galois, Bull. Soc. Math. Fr. 76 (1948) 114-155 (In French).

[10] D. A. Romano, Associated filters in quasi-ordered residuated systems, Contrib. Math. 1 (2020) 22-26.

[11] D. A. Romano, Filters in residuated relational system ordered under quasi-order, Bull. Int. Math. Virtual Inst. 10 (2020) 529-534.

[12] D. A. Romano, Comparative filters in quasi-ordered residuated system, Bull. Int. Math. Virtual Inst. 11 (2021) $177-184$.

[13] D. A. Romano, Implicative filters in quasi-ordered residuated system, Proyecciones 40 (2021) 417-424.

[14] D. A. Romano, Prime and irreducible filters in strong quasi-ordered residuated systems, Open J. Math. Sci., 5 (2021) $172-181$.

[15] D. A. Romano, Weak implicative filters in quasi-ordered residuated systems, Proyecciones 40 (2021) 797-804.

[16] A. Tarski, On the calculus of relations, J. Symb. Log. 6 (1941) 73-89.

[17] A. Tarski, S. Givant, A formalization of Set Theory Without Variables, Amer. Math. Soc., Providence, 1987. 